\title{
Double Pomeron Exchange: the early years
}

\author{
Michael G. Albrow \\ Fermi National Accelerator Laboratory, Batavia, IL 60510, USA
}

\begin{abstract}
I review the early experimental searches for double pomeron exchange, the first observations at the CERN Intersecting Storage Rings (ISR) and further studies at the SPS and Tevatron (fixed target). I only have space for a superficial coverage of some highlights, and will not cover later colliders with $\sqrt{s}>100 \mathrm{GeV}$.
\end{abstract}

Keywords: pomeron, diffraction, Central exclusive production PACS: $14.80 . \mathrm{Ec}, 27.75 . \mathrm{Dw}$

\section{INTRODUCTION}

In the late 1960's, before the introduction of QCD (Gross, Politzer and Wilczek showed that SU(3) is asymptotically free in 1973) hadronic interactions were mainly described in terms of Regge trajectories. Regge theory was based

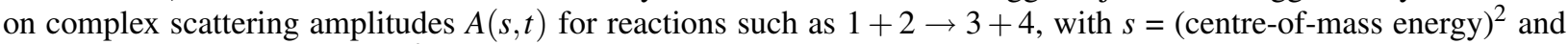

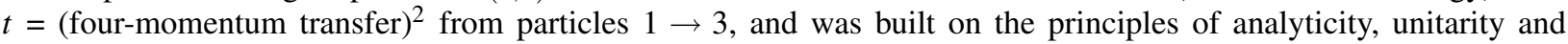
crossing symmetry. Although 4-momentum is transferred between the colliding particles, it is not carried by a particle (except for a virtual photon, $\gamma$, in Coulomb interactions) and is not emitted from one and absorbed by the other (that would be frame-dependent, reversible by a boost). In the centre-of-momentum frame of an elastic collision no energy is transferred, only 3-momentum; it is spacelike. The invariant 4-momentum exchange is carried by a sum of virtual states with the same quantum numbers, lying on a "Regge trajectory" with complex angular momentum $\alpha(t)$, approximately linear in $t$, which determines the behaviour of $A(s, t)$. The optical theorem relates the total cross section to the imaginary part of the $t=0$ scattering amplitude. This leads to a simple expression for the total $p p$ cross section, $\sigma_{t o t}=\mathbb{R} s^{\alpha_{\mathbb{R}}(0)-1}+\mathbb{P} s^{\alpha_{\mathbb{P}}(0)-1} \approx \mathbb{R} s^{-0.4525}+\mathbb{P} s^{0.0808}$ [1]. Two terms are needed because the total and elastic $p p$ cross sections fall with energy, flatten and then rise. The minimum, above which the IP term becomes dominant, happens at $\sqrt{s} \sim 20 \mathrm{GeV}$. The discovery that the total $p p$ cross section rises at the CERN ISR was the first clear evidence for the existence of the pomeron. It has the same quantum numbers as the vacuum. The Reggeon trajectory $\alpha_{\mathbb{R}}(t)$ is best measured with charge exchange reactions, such as $\pi^{-}+p \rightarrow \pi^{0}+n$ (which cannot yet be calculated in QCD) and when continued to positive $t \equiv M^{2}$ passes through integer values of $\alpha\left(M^{2}\right) \equiv J$ at the squaredmasses of known resonances ( $\rho, a_{2}$, etc.). The pomeron trajectory, which has intercept $\alpha_{\mathbb{P}}(t=0) \sim 1.08$ (see above), with a slope $\alpha^{\prime} \sim 0.2 \mathrm{GeV}^{-2}$ extrapolates to the next integer, $J=2$, at $M^{2} \sim 4 \mathrm{GeV}^{2}$ i.e. $M \sim 2 \mathrm{GeV}$, and should correspond to a tensor $(J=2)$ glueball state. The lightest glueball is expected to be a scalar $(J=0)$ and cannot be on the pomeron trajectory; its mass from lattice QCD calculations is expected to be $\sim 1600 \mathrm{MeV}$. Now, in 2014 , the glueball sector is still unclear, but both $f_{0}(1500)$ [2] and $f_{0}(1710)$ [3] are considered good candidates for a scalar glueball. Double pomeron exchange, DIPE, selects isoscalar states with even spin, positive parity and C-parity, and together with the glue-dominated nature of the pomeron, it provides an excellent channel for $J=0,2$ glueball searches [4]. Glue-dominated states with any quantum numbers can also be pair-produced in DIPE.

Experimental searches for DIPE started already in 1969 [5] in the Brookhaven 80" bubble chamber with $25 \mathrm{GeV} / c$ pions: $\pi^{-} p \rightarrow \pi^{-}+\left(\pi^{+} \pi^{-}\right)+p$. The centre-of-mass energy was very low, $\sqrt{s}=6.9 \mathrm{GeV}$, and they found 250 events with the characteristics of multi-Regge exchange but not DIPE; $\rho$ - and $\omega$-Reggeon exchanges dominated. Later bubble chamber searches [6], even with beams up to $205 \mathrm{GeV} / c$, did not succeed in making an observation of DIPE. The story is given in some more detail in Ref. [7]. Upper limits on the cross sections were $\sigma_{D P E}<44 \mu \mathrm{b}$; while a $20 \mu \mathrm{b}$ limit came from Ref. [8] using a $69 \mathrm{GeV} / c$ proton beam on a liquid hydrogen target with electronic detectors.

The CERN Intersecting Storage Rings, ISR, started in 1971 producing $p p$ collisions at much higher energies than any fixed target experiments (even today, being equivalent to $2.1 \mathrm{TeV} / c$ protons on a hydrogen target). In 1972 the Small Angle Spectrometer at the CERN ISR, at $\sqrt{s}=45 \mathrm{GeV}$ observed a peak at the Feynman variable $x_{F}=p_{z} / p_{\text {beam }} \gtrsim 0.95$ corresponding to high mass diffractive dissociation [9]. From kinematics, a proton with $x_{F}>$ 0.95 is adjacent to a rapidity gap $\Delta y>\ln \left(1 /\left(1-x_{F}\right)\right)=3$. A good "rule-of-thumb" for pomeron-dominance in an 

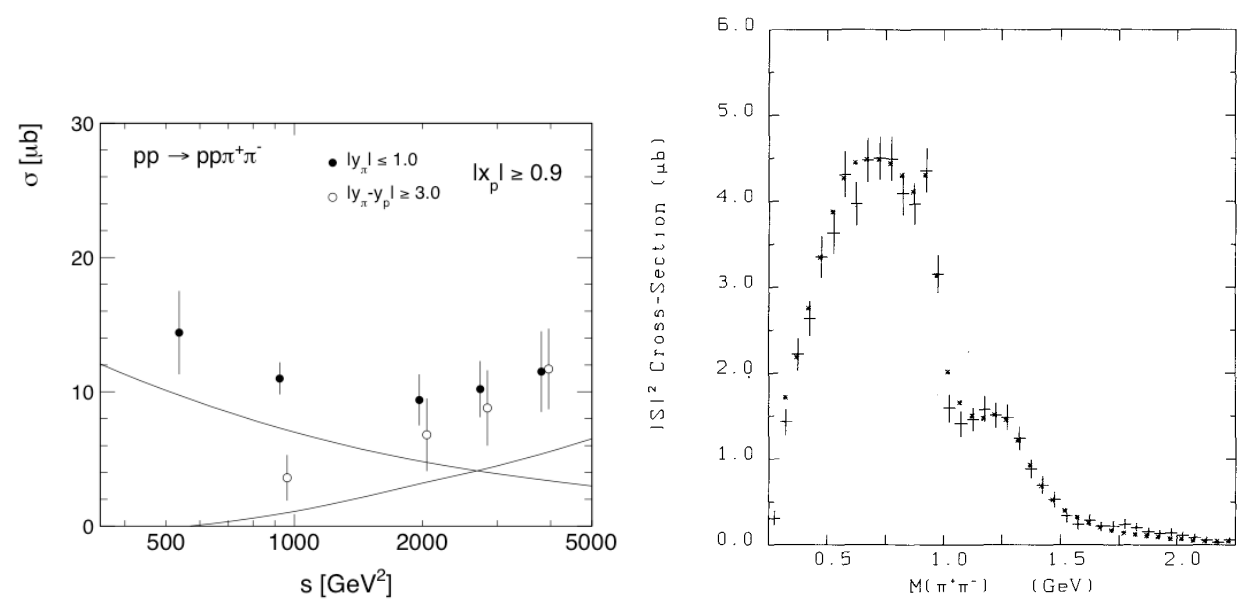

FIGURE 1. (left) Experimental results on $\sigma_{D P E}$ up to ISR energies, with Regge fits. (right) The central exclusive $\pi^{+} \pi^{-}$S-wave cross section distribution at $\sqrt{s}=63 \mathrm{GeV}$ (SFM).

inelastic interaction is to have a proton with $x_{F}>0.95$ or a rapidity gap $\Delta y>3$. The ISR data were fit to a tripleRegge model with a Reggeon, $\mathbb{R}$ or $\mathbb{P}$, coupled to the proton and interacting with the other proton with another Reggeon/pomeron exchange. A key theoretical issue was whether the triple-pomeron coupling $g_{\mathbb{P} \mathbb{P} I \mathbb{P}}$ vanishes at $t$ $=0$; the data showed otherwise. The phenomenology, with fits to the data, implied that one should have $\mathbb{P}+\mathbb{P} \rightarrow X$ reactions, i.e. DIPE, and revived interest in the process.

The first observation of DIPE came in 1976 [10] by experiment R407/408 at the Split Field Magnet (SFM) facility at the ISR. The SFM facility had a dipole field in the forward directions, but in the central region the field was complicated (with zero field at polar angle $\theta=90^{\circ}$, i.e. $\eta=0$ ). Clearly the study of DIPE with little background required colliding beams, ISR and above, and triggerable electronic detectors. This was reinforced by the last published hydrogen bubble chamber study [11] at Fermilab, with $147 \mathrm{GeV} / c \pi, K$, and $p$ beams, finding only 47 candidate events in 500,000 pictures, and quoting $\sigma \sim 20-50 \mu \mathrm{b}$. In the post-ISR years many excellent studies of central exclusive hadron production on a fixed target were done with $\sqrt{s}=13-29 \mathrm{GeV}$ using the Omega-spectrometer, with many different central states [12]. But some $\mathbb{R}+\mathbb{P}$ backgrounds were always present. The last fixed target DIPE experiment was E690 [13] at the Fermilab Tevatron with an $800 \mathrm{GeV} / c$ proton beam $(\sqrt{s}=40 \mathrm{GeV})$. Exclusive $X=\pi^{+} \pi^{-}, K_{s}^{0} K_{s}^{0}, K_{s}^{0} K^{ \pm} \pi^{\mp}$ and $\phi \phi$ channels were studied. The slow recoil proton was not measured, but was inferred from the missing mass squared of the event $\left(M_{m i s s}^{2} \sim m_{p}^{2}\right)$. A partial wave analysis (PWA) was made to select $S$-wave $(J=0)$ and $D$-wave $(J=2)$ intensities. The $S$-wave $\pi^{+} \pi^{-}$spectrum shape up to $2000 \mathrm{MeV}$ is essentially identical to that measured earlier at the ISR, with only a small D-wave $f_{2}(1270)$. However, if the fast proton has $p_{T}>1 \mathrm{GeV} / c$ the $f_{2}(1270)$ becomes more prominent. Much later data from CDF [14] showed a stronger $f_{2}(1270)$ signal, not detecting the leading protons but allowing dissociation. It seems that the DIPE spectra are different when the protons are detected at small $|t|$ and when only gaps are required; this could now be tested directly in CMS-TOTEM low-pileup runs at the LHC, by comparing central states with leading protons and with leading showers in the Forward Shower Counters, FSC.

Fig.1(left) shows CEP cross sections vs. $s$, fit to a falling $\mathbb{R}$ component and a rising $\mathbb{P}$ component, which dominates (in this kinematic region) only for $\sqrt{s} \gtrsim 50 \mathrm{GeV}$. If one instead selects $\left|x_{p}\right|>0.95$ the non-DIPE component is reduced and the DIPE signal emerges earlier.

Some ten years after the first observation of DIPE, more detailed studies from both the SFM and the Axial Field Spectrometer, AFS, were published. Breakstone et al. $[15,16]$ at $\sqrt{s}=45-63 \mathrm{GeV}$ at the SFM selected 4-track events with two leading particles, assumed to be protons, separated by rapidity gaps $\Delta y>3.0$ from a pair of pions each with $y_{\pi}<1.0$. A 4-C fit to $p+\pi^{+} \pi^{-}+p$ with $x_{F}(p)>0.9$ cleaned up the sample. They found $t_{1}$ and $t_{2}$ to be uncorrelated, and to have an exponential slope $b=-6.1 \mathrm{GeV}^{-2}$, half the elastic slope, for both $t_{1}, t_{2}$, and $\left(t_{1}+t_{2}\right)$, as expected for DIPE. The cross section is about $10 \mu \mathrm{b}$, showing some rise through this energy range [17]. The $M(\pi \pi)$ spectrum rises from threshold up to $1000 \mathrm{MeV}$, with no sign of a $\rho$-meson (forbidden in DIPE), and then drops rapidly, see Fig.1(right). This behavior is called a "cusp", occuring when the $K \bar{K}$ threshold opens, but the narrow $f_{0}(980)$ meson occurs at the same mass. A bump in the cross section looks like the $f_{0}(1270)$ state, but a partial wave analysis showed 
that the $J=2 \mathrm{D}$-wave is dominated there by S-wave, see Fig.1(right). This raises the question whether the data all the way up to $1500 \mathrm{MeV}$, where there is a break, are dominated by the $f_{0}(500) / \sigma$, a very broad $(\Gamma=(400-700) \mathrm{MeV})$ $I^{G} J^{P C}=0^{+} 0^{++}$(poorly understood) state, destructively interfering with the $f_{0}(980)$ to form a dip.

The AFS was designed for high- $E_{T}$ physics, with a uranium-scintillator calorimeter covering $\Delta \phi=2 \pi$, and codiscovered high- $E_{T}$ jets with UA2 at the $\operatorname{S} p \bar{p} \mathrm{~S}$ collider. To search for glueballs in DIPE, sets of drift chambers for proton tracking were added $[18,19]$ along the beam pipes, with veto counters covering $1.5<|\eta|<3$. Events kinematically compatible with $p+h^{+} h^{-}+p$ with $x_{p}>0.95$ were selected, and the central hadrons were identified by ionisation, $\frac{d E}{d x}$. At $\sqrt{s}=63 \mathrm{GeV}$ there were $87,000 \pi^{+} \pi^{-}, 523 K^{+} K^{-}$, and $64 p \bar{p}$ events, with a small amount of data at $\sqrt{s}=45 \mathrm{GeV}$. The general features are similar to those in Fig.1(right), including S-wave dominance up to about $1500 \mathrm{MeV}$, apart from a small $f_{0}(1270)$. The only established [20] scalar meson in this region is the broad $f_{0}(1300)$. With higher statistics, the data extend to $3500 \mathrm{MeV}$, showing a broad bump from 1500 to $2500 \mathrm{MeV}$.

The ISR also provided $\alpha-\alpha$ collisions at $\sqrt{s}=126 \mathrm{GeV}$, and both the AFS and the CERN-Naples-Pisa-Stony Brook experiment [21] measured $\alpha+\pi^{+} \pi^{-}+\alpha$ events, clearly coherent as the $\alpha$ stay intact while pions are created. The mass spectrum has the same shape as in $p p$, within the large statistical uncertainty, the $t$-slope is about half that of elastic $\alpha \alpha$ scattering, and $\sigma$ (DIPE) is about a factor $2 \times$ higher.

The SPS fixed target studies with the $\Omega$-spectrometer provided a wealth of information, but at too low $\sqrt{s}$ for DIPE dominance. Our goal should be to get much higher statistics (e.g. $10^{6}$ events/channel) with both protons measured at high $\sqrt{s}$, e.g. at RHIC or the LHC, and in many channels with identified hadrons including (but not only) $K^{+} K^{-}$, $K_{S}^{0} K_{S}^{0}, \phi \phi, \eta \eta, \pi \pi K K$. This could actually be done in about a week of low pileup running at the LHC. If there is, as expected in QCD, a scalar glueball with mass $>1000 \mathrm{MeV}$ it will probably be quite wide and therefore have such a short lifetime that if produced inclusively it will decay within the hadron size $\sim 1 \mathrm{fm}$. It will not be an isolated hadron, but live and die in a "messy" environment. Only in direct DIPE production will it be alone, in a clean (in fact, vacuum) environment. This is diffractive excitation of virtual strongly interacting states in the vacuum! Interesting!

\section{REFERENCES}

1. See e.g., V. Barone and E. Predazzi, High Energy Particle Diffraction, Springer (2002).

2. See e.g., W. Ochs, The status of glueballs, J. Phys. G 40, 043001 (2013).

3. S. Janowski, F. Giacosa, D.H. Rishke, Is $f_{0}(1710)$ a glueball?, arXiv:1408:4921 (2014).

4. F.E. Low, Phys. Rev. D 12, 163 (1795); S. Nussinov, Phys. Rev. 34, 1286 (1975).

5. R. Lipes, G. Zweig, and W. Robertson, Phys. Rev. Lett. 22, 433 (1969).

6. M. Derrick et al., Phys. Rev. Lett. 32, 80 (1974).

7. M.G. Albrow, Int. J. Mod. Phys A. 29, 1446014 (2014).

8. D. Denegri et al., Nucl. Phys. B98, 189 (1975).

9. M.G. Albrow et al., Nucl. Phys. B108, 1 (1976).

10. M. Della Negra et al., Phys. Lett. 65B, 394 (1976).

11. D. Brick et al., FERMILAB-PUB-80-112E (1980).

12. A. Kirk, Int. J. Mod. Phys A. 29, 1446001 (2014) and references therein.

13. G. Gutierrez and M.A. Reyes, Int. J. Mod. Phys A. 29, 1446008 (2014) and references therein.

14. M.G. Albrow, Int. J. Mod. Phys A. 29, 1446009 (2014) and references therein.

15. A. Breakstone et al., Z. Phys. C 31, 185 (1986).

16. H.G. Fischer, W. Geist, and M. Makariev, Int. J. Mod. Phys A. 29, 1446005 (2014).

17. M.G. Albrow, T.D. Coughlin, and J.R. Forshaw, Prog. Part. and Nucl. Phys. 65, 149 (2010).

18. T. Åkesson et al. (AFS Collaboration), Phys. Lett. 133B, 268 (1983).

19. T. Åkesson et al. (AFS Collaboration), Nucl. Phys. B 264, 154 (1986).

20. K.A. Olive et al. (Particle Data Group), Chin. Phys. C 38, 090001 (2014).

21. V. Cavasinni et al., Z. Phys. C 28, 487 (1983). 